\title{
Arresting doubly excited electronic state mediating lightning speed ring-opening reaction of 1,3- cyclohexadiene
}

\section{Shutaro Karashima}

Kyoto University

\section{Ryuta Uenishi}

Kyoto University

\section{Takuya Horio}

Kyoto University

\section{Manabu Kanno}

Tohoku University https://orcid.org/0000-0003-4701-906X

\author{
Alexander Humeniuk \\ Universität Würzburg \\ Roland Mitric \\ University of Würzburg

\section{Tetsuro Ohta} \\ Kyoto University \\ Junichi Nishitani \\ Kyoto University \\ Toshinori Suzuki ( $\nabla$ suzuki@kuchem.kyoto-u.ac.jp ) \\ Kyoto University https://orcid.org/0000-0002-4603-9168
}

\section{Article}

Keywords: 1,3-cyclohexadiene, 1,3,5-hexatriene, stereochemical reactions

Posted Date: August 13th, 2020

DOl: https://doi.org/10.21203/rs.3.rs-53810/v1

License: (1) (i) This work is licensed under a Creative Commons Attribution 4.0 International License.

Read Full License 


\title{
Arresting doubly excited electronic state mediating lightning speed ring-opening reaction of 1,3-cyclohexadiene
}

\author{
Shutaro Karashima, ${ }^{1}$ Ryuta Uenishi, ${ }^{1}$ Takuya Horio, ${ }^{1 \dagger}$ Manabu Kanno, ${ }^{2}$ Alexander Humeniuk, ${ }^{3}$ \\ Roland Mitrić, ${ }^{3}$ Tetsuro Ohta, ${ }^{1}$ Junichi Nishitani, ${ }^{1}$ and Toshinori Suzuki ${ }^{1 *}$ \\ ${ }^{1}$ Department of Chemistry, Graduate School of Science, Kyoto University, Kyoto 606-8502, Japan \\ ${ }^{2}$ Department of Chemistry, Graduate School of Science, Tohoku University, Sendai 980-8578, Japan \\ ${ }^{3}$ Universität Würzburg, Institut für Physikalische und Theoretische Chemie, Emil-Fischer-Str. 42, \\ 97074 Würzburg, Germany
}

\begin{abstract}
The photoinduced ring-opening reaction of 1,3-cyclohexadiene to produce 1,3,5-hexatriene is a wellknown example of the Woodward-Hoffmann rule for stereochemical reactions governed by molecular orbital symmetry, and it plays an essential role in photobiological synthesis of vitamin $\mathrm{D}_{3}$ in the skin. Since the photoexcited $1^{1} \mathrm{~B}$ state of 1,3 -cyclohexadiene is not electronically correlated to the ground state of 1,3,5-hexatriene, the reaction is expected to proceed via non-adiabatic transitions through a doubly excited $2^{1} \mathrm{~A}$ electronic state. However, spectroscopic observation of this elusive state has been difficult. Here we present the results of a photoelectron spectroscopy study using table-top ultrafast deep and extreme UV lasers, based on filamentation four-wave mixing and high-harmonic generation, which enabled us to arrest the $2^{1} \mathrm{~A}$ state. It is shown that the ring-opening reaction takes only 60 fs to complete, which is a considerably shorter time than previous experimental and theoretical estimates. The ballistic reaction creates vibrational coherence in the reaction products.
\end{abstract}




\section{Introduction}

The photoinduced ring-opening reaction of 1,3-cyclohexadiene (CHD) to produce 1,3,5hexatriene (HT) is one of the best-known examples of electrocyclic chemical reactions, ${ }^{1,2}$ and it plays a central role in photobiological synthesis of vitamin $\mathrm{D}_{3}$ in the skin by facilitating photoisomerization of 7-dehydrochoestrerol to previtamin $\mathrm{D}_{3}{ }^{3}$ The ring-opening reaction of CHD follows the WoodwardHoffmann rule, and $\mathrm{C}_{5}-\mathrm{C}_{6}$ bond rupture via the electronically excited state occurs with conrotatory motion of the end $\mathrm{CH}_{2}$ groups. ${ }^{4}$ The reaction starts with a HOMO (12a)-LUMO (11b) transition in CHD induced by ultraviolet (UV) radiation, and the photoexcited $1^{1} \mathrm{~B}$ state ultimately relaxes to the ground electronic state $\left(1^{1} \mathrm{~A}\right),{ }^{5}$ during which the system bifurcates into HT (a ring-opening reaction) and CHD (non-reactive internal conversion). However, it should be noted that the photoexcited $1^{1} \mathrm{~B}$ state is not electronically correlated with the ground state of HT. Non-adiabatic transition(s) among the electronic states must be involved in order to facilitate the reaction. Van der Lugt and Oosterhoff performed a theoretical analysis of a related ring-closing reaction from butadiene to cyclobutene, and they pointed out that the reaction is mediated by a two-electron excited state. ${ }^{6}$ The same mechanism is also expected here, as illustrated in Fig. 1 . The potential energy surface of the $1^{1} \mathrm{~B}$ state undergoes a conical intersection (CoIn) with that of the doubly excited $2^{1} \mathrm{~A}$ state to induce a non-adiabatic transition, and the $2^{1} \mathrm{~A}$ state mediates ultrafast internal conversion to the ground state to induce the ring-opening reaction. The essential feature of the electronic structure of this system is that the energy ordering of the HOMO and LUMO is reversed between the reactant and product, which places the $1{ }^{1} \mathrm{~B}, 2^{1} \mathrm{~A}$, and $1^{1} \mathrm{~A}$ states in energetic proximity. Thus, it is the doubly excited $2^{1} \mathrm{~A}$ state that plays a key role in the reaction; however, its spectroscopic detection has been difficult. (The $\mathrm{C}_{2}$ symmetry of CHD is lifted during the course of the reaction; however, we refer to the relevant electronic states and molecular orbitals using the symmetry notation under the $\mathrm{C}_{2}$ point group throughout this paper.)

Ultrafast photoionization mass spectrometry by $\mathrm{Fu} ß$ and coworkers reported decades ago $5,7,8$ inspired a number of subsequent studies on this benchmark system. 5, 7, 8, 9, 10,11, 12, 13, 14, 15, 16, 17, 18, 19, 20, 21, 22, 23, 24, 25, 26, $27 \mathrm{Fuß}$ et al. employed strong field ionization of the transient species using $800 \mathrm{~nm}$ pulses, and suggested the wave packet propagation time on the $1^{1} \mathrm{~B}$ surface, the time for transition from the $1^{1} \mathrm{~B}$ to $2^{1} \mathrm{~A}$ states, and the lifetime of the $2^{1} \mathrm{~A}$ state to be 21,35 and $80 \mathrm{fs}$, respectively. Although the claims by Fuß et al. were intriguing, definitive spectroscopic assignments of the ionized species and electronic states were lacking in their mass spectrometric study, leaving a large degree of ambiguity in their arguments. The reaction has been studied more recently using photoelectron spectroscopy, ${ }^{20,22,23,25}$ X-ray absorption spectroscopy ${ }^{26}$ and electron diffraction; ${ }^{27}$ however, the time resolution (120 -160 fs) of these experiments was insufficient for capturing the electronic dynamics in 
real time. Meanwhile, computational studies provided a great deal of mechanistic insights into the reaction; however, the calculated reaction times were scattered, falling in the range of 70-280 fs.

The objective of this study is to experimentally identify non-adiabatic transitions via the doubly excited state of CHD and establish the mechanistic picture of this paradigmatic photoinduced ring-opening reaction. To this end, we employ ultrafast photoelectron spectroscopy using deep UV (DUV; $267.5 \mathrm{~nm}, 4.6 \mathrm{eV}, 28 \mathrm{fs}$ ) pump and extreme UV (XUV; $57 \mathrm{~nm}, 21.7 \mathrm{eV}, 43 \mathrm{fs}$ ) probe pulses. ${ }^{28}$ Since XUV radiation ionizes all chemical species involved in the reaction, XUV photoelectron spectroscopy enables complete observation from the Franck-Condon region in the $1^{1} \mathrm{~B}$ state of CHD until the final states of the reaction products. While XUV radiation produced by high-harmonic generation $(\mathrm{HHG})^{29,30,31,32}$ has been employed in ultrafast spectroscopy; ${ }^{32,33,34}$ experiments with DUV pump pulses are scarce owing to the technical difficulties in generating ultrashort DUV pulses. ${ }^{35}$ In the present study, we combined a filamentation four-wave mixing (FFWM) DUV light source ${ }^{36}$ and a HHG XUV light source for the first time to achieve the time-resolution of $48 \pm 2$ fs.

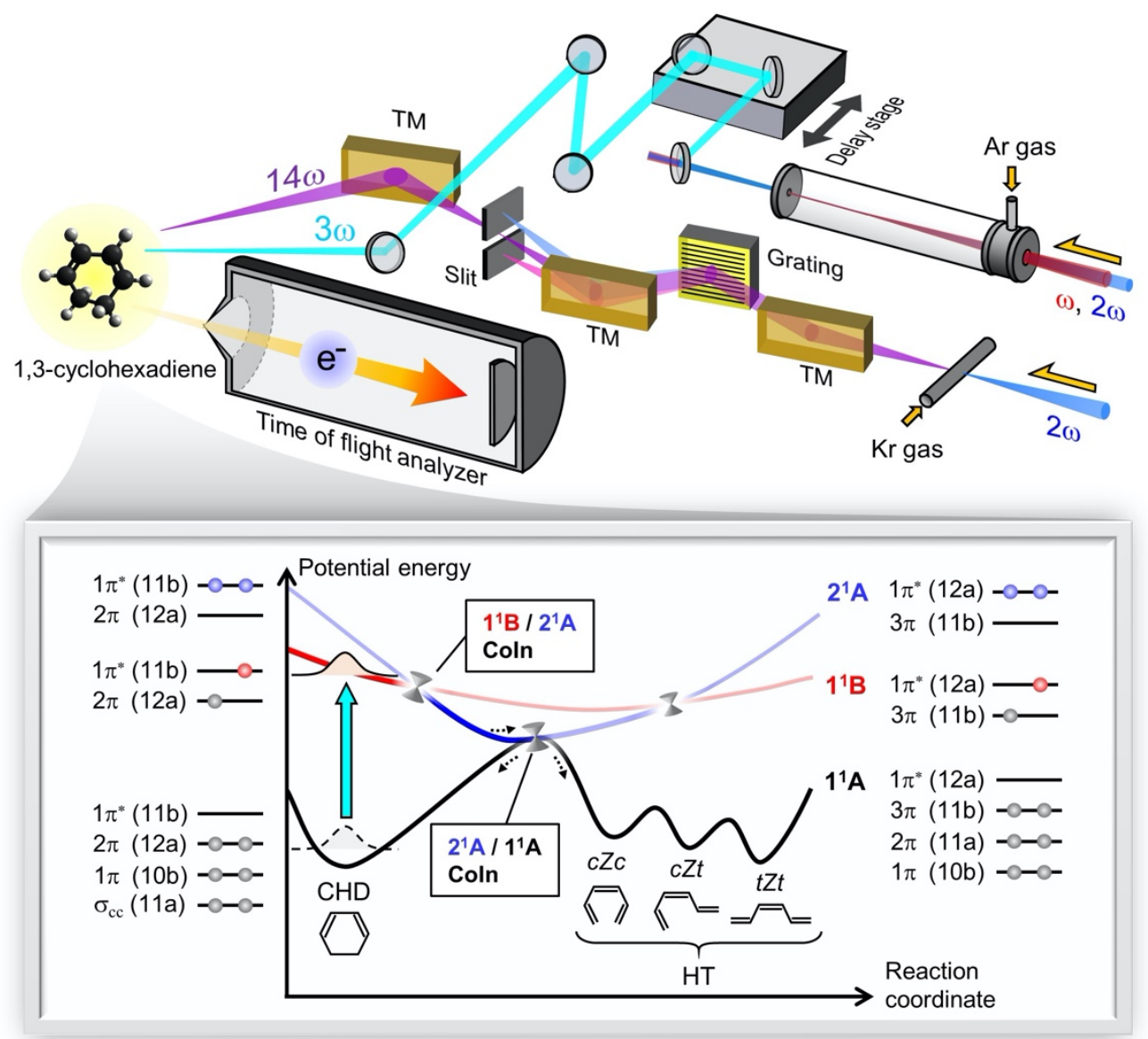

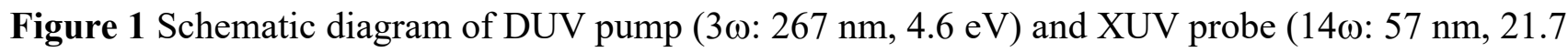
eV) experiment. (TM: toroidal mirror, CoIn: conical intersection, CHD: 1,3-cyclohexadiene, HT: 1,3,5-hexatriene) 


\section{Results and Discussion}

Figure 2 shows photoelectron spectra measured as a function of the pump-probe delay time. The pump pulses excite CHD to the $1{ }^{1} \mathrm{~B}$ state, and the probe pulses induce photoemission from all transient species in all electronic states. The spectra are plotted against the electron binding energy (eBE), given by the difference between the XUV photon energy and the measured electron kinetic energy (eKE). Since the XUV probe pulses cause photoemission from the ground electronic state of CHD for all delay times, its contribution has already been subtracted from the pump-probe spectra. The three negative-intensity bands indicated in violet in Fig. 2 are the ground-state bleach (depopulation) induced by the pump pulses. We estimate that the ground-state bleach (the excitation efficiency) is $1.8 \%$ under our experimental conditions. The positive signals at short pump-probe delay times $(<100 \mathrm{fs})$ are from the excited electronic state of CHD, while those at longer delay times are from reaction products. The ground-state bleach signal is never recovered, because the reaction products show different photoelectron spectra. In Fig. 2, it can be seen that the spectral features of the positive intensity bands, shown in green to red, rapidly change within $100 \mathrm{fs}$ and become almost constant thereafter. This clearly indicates that the ring-opening reaction is completed in less than 100 fs. It can also be seen that the photoelectron signal intensities exhibit oscillations, most notably at around 8 and $10 \mathrm{eV}$. These are vibrational quantum beats of the reaction products observed for the first time for this reaction. 

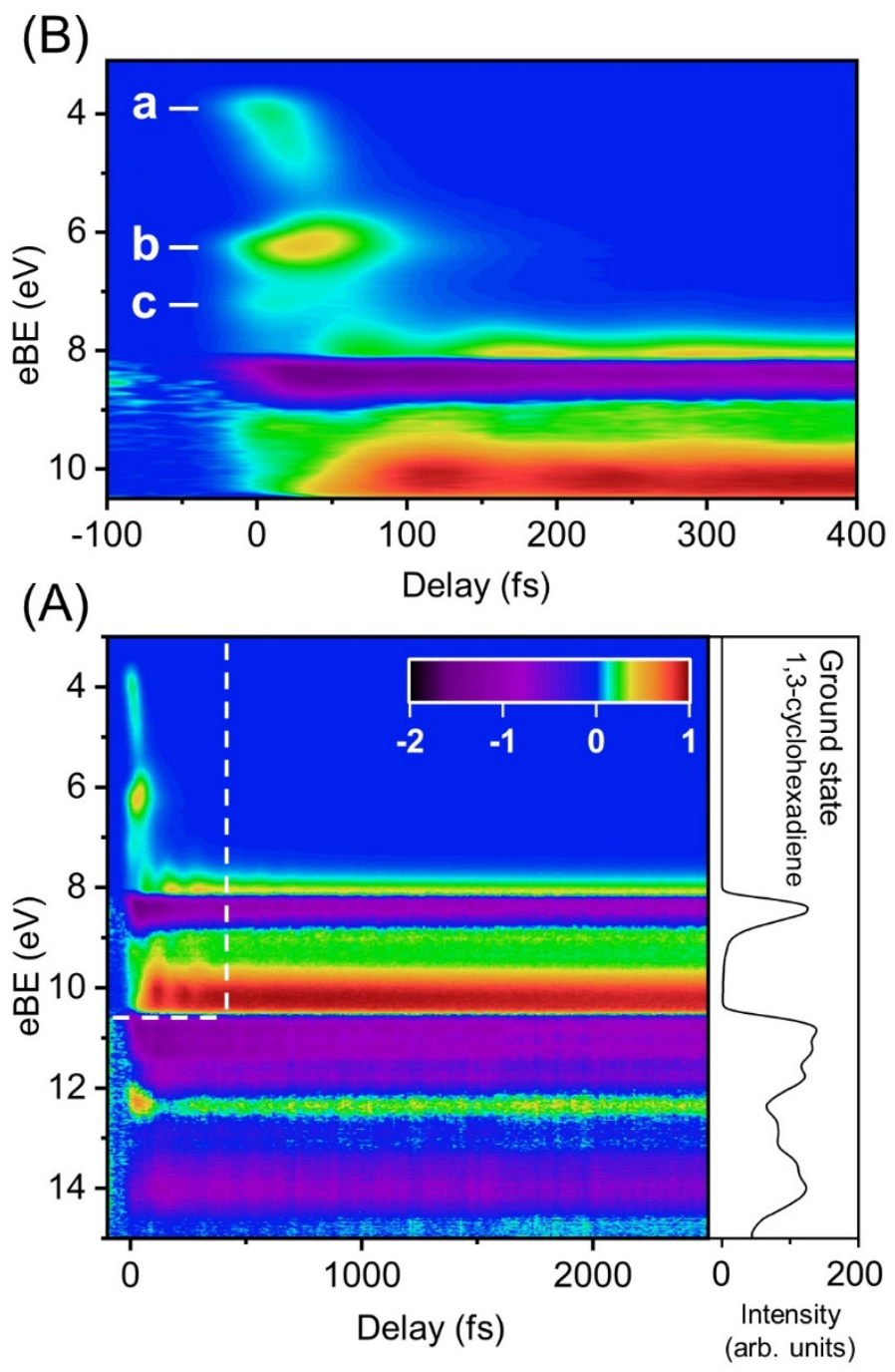

Figure 2 (A) Two-dimensional map of the photoelectron spectra measured as a function of the pumpprobe delay time, with the photoelectron spectrum of the ground-state CHD shown on the right. The one-color photoionization signal from unexcited CHD has already been subtracted; therefore, the negative signals shown in violet correspond to signals due to photoexcitation depletion by the pump pulses. (B) Blowup of the map at short delay times. Photoionization from the $1^{1} \mathrm{~B}$ to the cationic ground state $1^{2} \mathrm{~A}$ and the $1^{2} \mathrm{~B}$ and $2^{2} \mathrm{~B}$ (or $3^{2} \mathrm{~B}$ ) excited states is indicated by $\mathrm{a}, \mathrm{b}$ and $\mathrm{c}$, respectively.

As shown in Fig. 1, the $1^{1} \mathrm{~B}$ state has a leading electron configuration of $\left[(10 b)^{2}(12 a)^{1}(11 b)^{1}\right]$ created by the HOMO-LUMO transition. (The notations in the square brackets are the relevant molecular orbitals and their occupancies.) In Fig. 2(B), the photoelectron signal from the $1^{1} \mathrm{~B}$ state at time zero appears at three eBE values of 3.75, 6.2 and $7.0 \mathrm{eV}$ (indicated by a, b and c, respectively), which correspond to ionization to the $1^{2} \mathrm{~A}\left[(10 b)^{2}(12 a)^{1}(11 b)^{0}\right], 1^{2} \mathrm{~B}\left[(10 b)^{1}(12 a)^{2}(11 b)^{0}\right]$ and $2^{2} \mathrm{~B}$ (or 
$\left.3^{2} \mathrm{~B}\right)\left[(10 b)^{2}(12 a)^{0}(11 b)^{1}\right]$ cationic states. The $1^{2} \mathrm{~A} \leftarrow 1^{1} \mathrm{~B}$ band rapidly shifts from 3.75 to $5.25 \mathrm{eV}$ within $30 \mathrm{fs}$, because the $1{ }^{1} \mathrm{~B}$ potential energy surface has a steep gradient for the $\mathrm{C}_{5}-\mathrm{C}_{6}$ stretching coordinate, while the $1^{2} \mathrm{~A}$ cationic surface has a deep potential well. Therefore, eBE continuously increases as the vibrational wave packet propagates in the descending direction of the $1^{1} \mathrm{~B}$ surface from the FranckCondon region. The intensity of the b-band is initially weaker than that of the a- and c-bands owing to the electron configuration of $1^{2} \mathrm{~B}\left[(10 b)^{1}(12 a)^{2}(11 b)^{0}\right]$ unfavorable for one-photon transitions from the $1^{1} \mathrm{~B}$ state. However, the b-band intensity grows with time and then exhibits a maximum, which is attributed to emergence of the $1^{2} \mathrm{~A} \leftarrow 2{ }^{1} \mathrm{~A}$ photoionization process. Quantum chemical calculations using XMS-CASPT2 (extended multi-state complete active space second-order perturbation theory) ${ }^{37}$ estimate the $\mathrm{eBE}$ of the $2^{1} \mathrm{~A}$ state in its equilibrium geometry to be $6.2 \mathrm{eV}$, which supports our assignment of the $1^{2} \mathrm{~A} \leftarrow 2^{1} \mathrm{~A}$ band.

We performed global fitting of the observed spectra to separate the contributions of different ionization channels, as shown in Figs. 3(A) and 3(B) (see Supplementary Information for the details). Figure 3(C) shows the integrated band intensities as a function of time, in which one can see that the ionization signal from the $2^{1} \mathrm{~A}$ state is delayed with respect to that from the $1^{1} \mathrm{~B}$ state. We constructed a kinetic model to explain these experimental data. After testing several kinetic schemes, we found that the one shown in Fig. 3(E) explains the results well. The model indicates that the photoexcited wave packet created in the $1^{1} \mathrm{~B}$ state propagates along the descending potential slope and reaches the $1^{1} \mathrm{~B}-2^{1} \mathrm{~A}$ CoIn region at a delay time centered at $32 \pm 1 \mathrm{fs}$, and $88 \pm 3 \%$ of these non-stationary states change their electronic character from $1^{1} \mathrm{~B}$ to $2^{1} \mathrm{~A}$. The remaining $12 \%$ retain their $1^{1} \mathrm{~B}$ electronic character and eventually undergo exponential decay to the $2^{1} \mathrm{~A}$ state with a time constant of $60 \pm 23$ fs. This $12 \%$ of excited states with a longer residence time are evident in Figs. 2, 3(A) and 3(B) as a weak photoelectron signal tailing after the main peak. The lifetime of the $2^{1} \mathrm{~A}$ state is only $23 \pm 1 \mathrm{fs}$. Thus, a clear picture of the wave packet dynamics emerged. The photoexcited wave packet reaches the $1^{1} \mathrm{~B}$ $2^{1} \mathrm{~A}$ CoIn after about $32 \mathrm{fs}$, and $88 \%$ follows an adiabatic reaction path in the avoided crossing region, and only $12 \%$ make a non-adiabatic transition to the upper surface to maintain the ${ }^{1} \mathrm{~B}$ character. The wave packet on the $2^{1} \mathrm{~A}$ surface rapidly proceeds to the $2^{1} \mathrm{~A}-1^{1} \mathrm{~A}$ CoIn to undergo ultrafast internal conversion to the ground state without wandering around the (pericyclic) minimum on the $2^{1} \mathrm{~A}$ potential energy surface. Consequently, the overall lifetime in the excited state is as short as $55(32+23)$ fs for the majority ( $88 \%$ ) of the non-stationary states. The average reaction time via the adiabatic and non-adiabatic excited-state paths is $62 \mathrm{fs}(0.88 \times 55 \mathrm{fs}+0.12 \times 115 \mathrm{fs})$. The excited-state lifetime of 60 fs is drastically shorter than the values so far reported. Fig. 3(D) illustrates the populations in the $1^{1} \mathrm{~B}$, $2^{1} \mathrm{~A}$ and $1^{1} \mathrm{~A}$ states without convoluting with the experimental time resolution. 
Our model assumes that the population decay starts immediately after the system changes to the $2^{1} \mathrm{~A}$ electronic state. In reality, the wave packet is expected to propagate on the $2^{1} \mathrm{~A}$ surface for a finite time to arrive at the $2^{1} \mathrm{~A}-1^{1} \mathrm{~A}$ CoIn region and start decaying to the $1^{1} \mathrm{~A}$ state. Therefore, it is likely that there is a time lag between the arrival time of the wave packet at the former and the latter CoIn. Thus, 32 fs is the upper bound for the arrival time of the wave packet at the first CoIn.
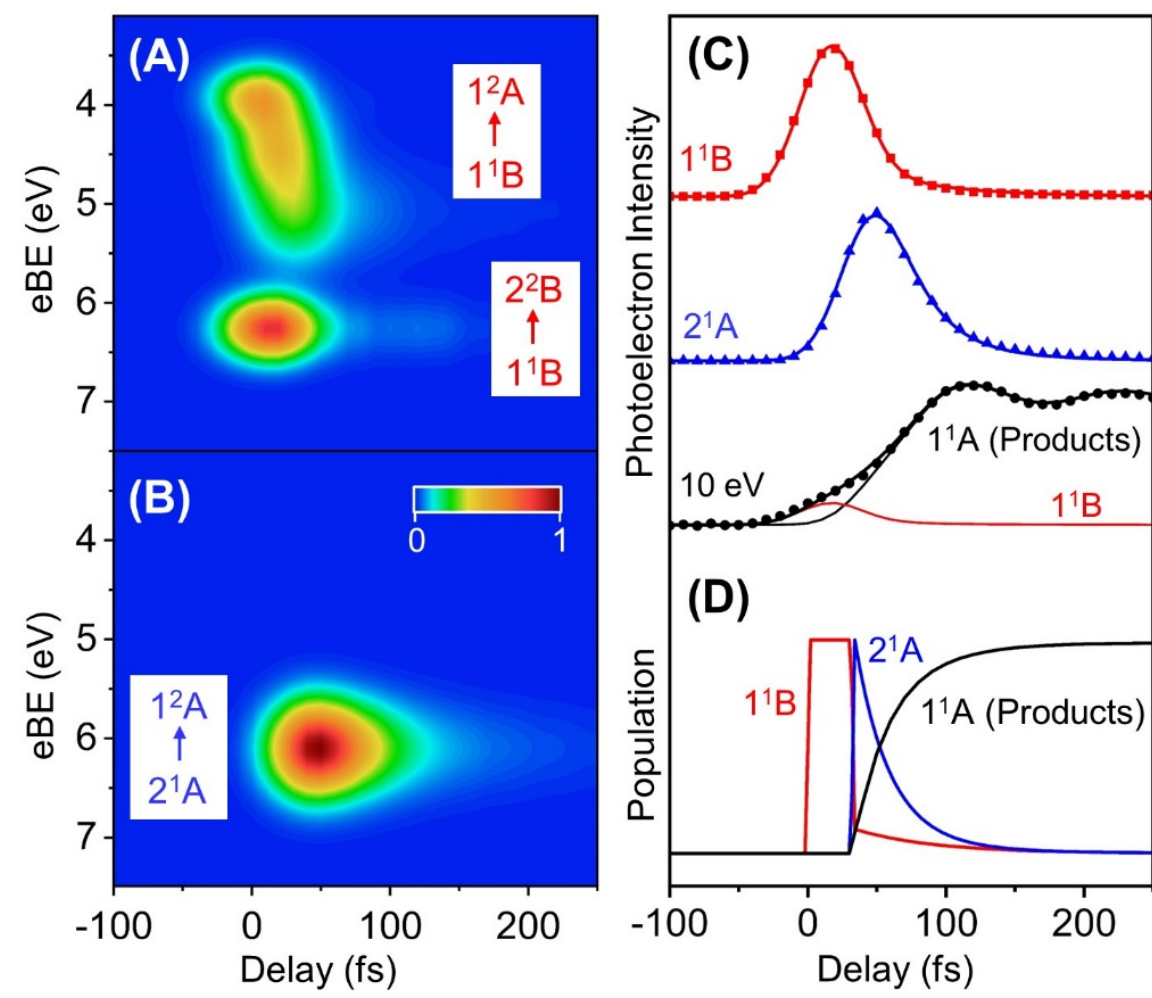

(E)

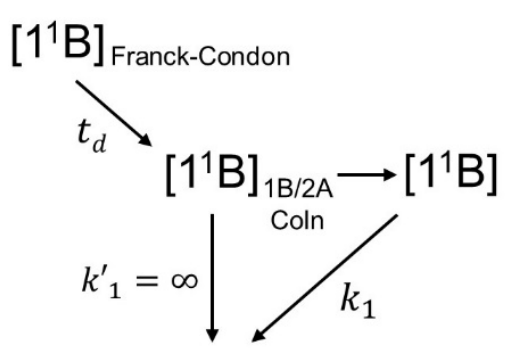

$\left[2^{1} \mathrm{~A}\right]$

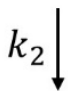

$\left[1^{1} \mathrm{~A}\right]$

Figure 3 Photoelectron spectra of (A) $1^{1} \mathrm{~B}$ and (B) $2^{1} \mathrm{~A}$ separated using least-squares fitting with the kinetics scheme shown in (E). (C) Time profiles of photoelectron intensities. Dots and solid lines show the experimental data and fitting results, respectively. Red and blue represent ionization from the $1^{1} \mathrm{~B}$ and $2^{1} \mathrm{~A}$ states, respectively, while black represents the integrated photoelectron intensity in the eBE range of 9.9-10.0 eV, which is predominantly due to reaction products. (D) Population in each electronic state calculated by deconvoluting with the experimental time resolution. (E) Kinetic scheme employed for the analysis. Bifurcation of the dynamics via non-adiabatic transitions at the $1{ }^{1} \mathrm{~B} / 2^{1} \mathrm{~A}$ conical intersection is taken into account. See Supporting Information for more details of our analysis.

Each transient photoelectron spectrum measured at a given delay time is the sum of negative and positive signals. For example, the spectrum for a short time delay of less than $30 \mathrm{fs}$ consists of a 
positive signal from the excited state of CHD and a negative signal from the depleted ground state of CHD, $\Delta S\left(t_{1}\right)=S($ Excited State $)-S($ CHD $)$, while the spectrum for a long delay time consists of a positive signal from the reaction products and a negative signal from the depleted ground state of CHD, $\Delta S\left(t_{2}\right)=S$ (Products) $-S($ CHD $)$. Therefore, we took the difference between the spectra at $30 \mathrm{fs}$ and $2.5 \mathrm{ps}, \Delta S(2.5 p s)-\Delta S(30 f s)=S$ (Products) $-S($ Excited State $)$, in order to cancel $S($ CHD $)$, and obtained the spectrum shown in Fig. 4(A). In this spectrum, the negative signal in the eBE region below $8 \mathrm{eV}$ is primarily due to the excited state of $\mathrm{CHD}$, whereas the positive signal at $8-10 \mathrm{eV}$ is from the reaction products. Above $11 \mathrm{eV}$, ionization to a dense cationic excited state manifold occurs from both the excited states of CHD and the reaction products, and these negative and positive signals largely cancel each other. When we compare the observed reaction-product spectrum with those of cold CHD and HT, we find that the reaction product spectrum is more like the HT spectrum. However, it is noted that the reaction products are highly vibrationally excited, as the pump photon energy is entirely converted into the internal energy of the reaction products $(4.6 \mathrm{eV}$ for $\mathrm{CHD}$ and $3.5-3.8 \mathrm{eV}$ for HT), and the energy cannot dissipate in the gas phase via intermolecular interactions. Thus, we performed semi-empirical dynamical calculations to predict the photoelectron spectra of vibrationally excited CHD and HT products at 1 ps after photoexcitation (See Supplementary Information for details). As shown in Fig. 4(A), the predicted spectra of CHD and HT are very similar to each other, and their differentiation in the photoelectron spectra is difficult. Thus, with our best efforts using ultrafast photoelectron spectroscopy, it is currently difficult to differentiate CHD and HT and estimate the reaction product branching ratio. Currently, the most common theoretical estimate for the CHD/HT branching ratio in the gas phase is $1: 1$, but this is yet to be experimentally verified.

As mentioned earlier, there are periodic intensity oscillations in the photoelectron spectra, which are ascribed to vibrational quantum beats in the reaction products. These oscillatory features are observed only in a narrow eBE window, where no strong signal occurs from cold CHD. It is noted that all transient signals arise from only $1.8 \%$ of CHD molecules photoexcited to the $1^{1} \mathrm{~B}$ state, and that a minute fluctuation of the photoelectron signal from unexcited cold CHD $(98.2 \%$ remaining in the 
ground state) obscures the weak quantum beat signals from the reaction products. Figure 4(B) shows photoelectron time profiles measured at an eBE of 8 and $10 \mathrm{eV}$, and the extracted oscillatory components. The two profiles show some interesting differences. One is that the quantum beat persists for a longer time period at $8 \mathrm{eV}$, and the other is that the phase of the oscillations is shifted by $\pi$ between the two profiles. On the other hand, Fourier transforms of the oscillatory components shown in Fig. 4(C) indicate that the beat frequencies are similar for the two traces. In ultrafast spectroscopy, impulsive vibrational Raman scattering by pump pulses creates vibrational coherence in the ground state of the reactant, which must be differentiated from the coherence in the reaction products. In the present case, the following features suggest that the observed vibrational quantum beats are attributed to the reaction products. Firstly, the eBE region (for example, $9-10 \mathrm{eV}$ ) where the beat appears is well separated from eBE of the ground-state CHD. Secondly, the beat intensity increases with increasing reaction product signal. And, finally the observed beat frequencies differ from the fundamental vibrational frequencies known for CHD. ${ }^{38}$

Previously, vibrational coherence in the photochemical reaction product has been observed for the cis-trans photoisomerization of rhodopsin that occurs in $30 \mathrm{fs} .{ }^{39}$ The phenomenon was indicated by the pioneering Raman spectroscopy studies by Mathies and coworkers ${ }^{40,41,42,43}$ and unambiguously shown by the recent ultrafast transient grating spectroscopy study by Miller and coworkers. ${ }^{39}$ The ringopening reaction of CHD has similar strong electronic coupling and an extremely short reaction time. ${ }^{44}$ The 48-fs time resolution in our experiment provided a narrow frequency window for observation of vibrational coherence, but experiments with a higher time resolution will enable further examination of the vibrational dynamics in this paradigmatic reaction system.

In conclusion, we performed ultrafast photoelectron spectroscopy to examine a pericyclic ring-opening reaction of CHD using a deep UV ultrafast laser with filamentation four-wave mixing and an XUV ultrafast laser with high-harmonic generation. We unambiguously identified the formation and decay of the doubly excited $2^{1} \mathrm{~A}$ electronic state that plays a key role in the reaction. The overall reaction time of $60 \mathrm{fs}$ is strikingly shorter than previous estimates, and illustrates the ballistic nature 
of this reaction. Vibrational wave packet motion was observed for the first time for the reaction products.
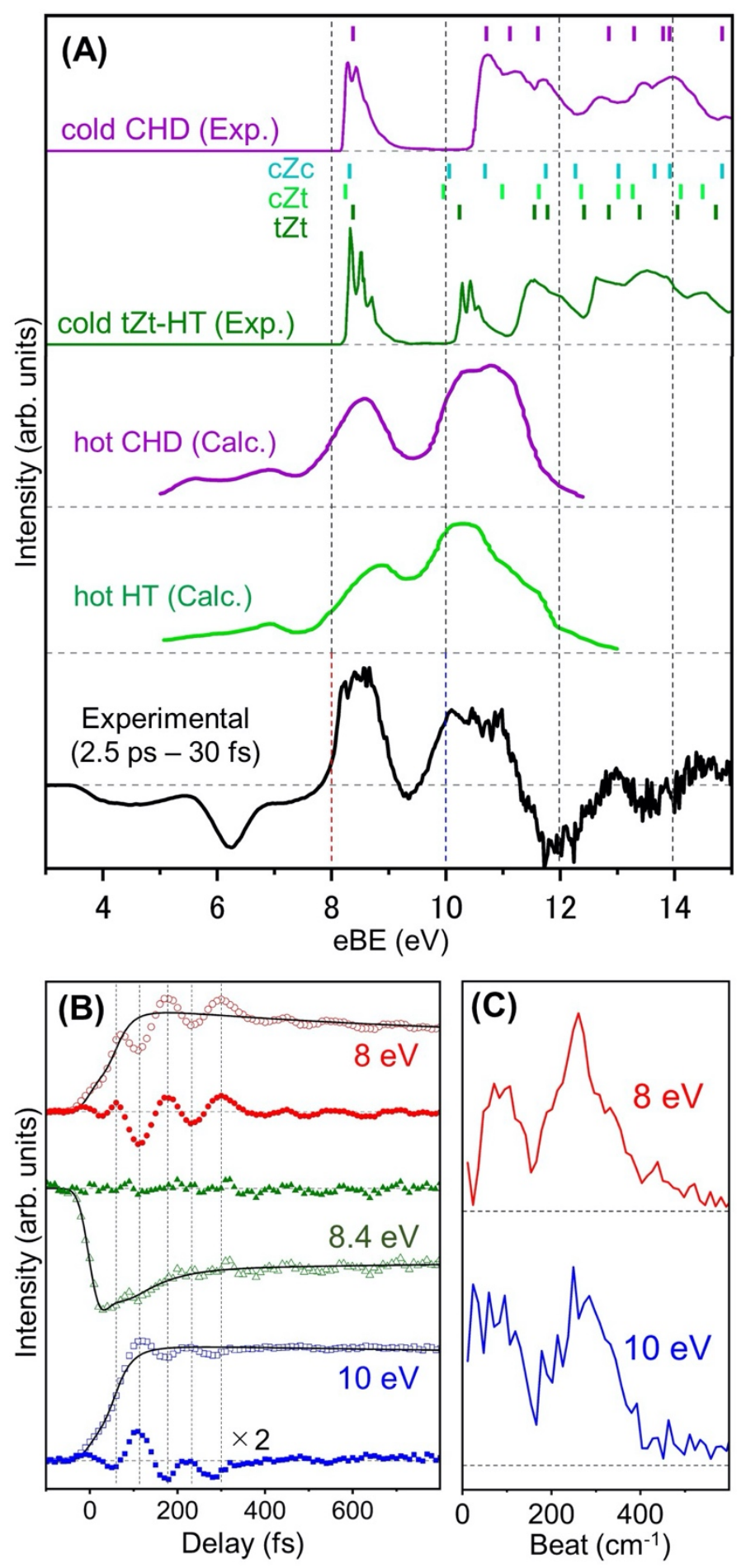

Figure 4 (A) Difference between transient photoelectron spectra measured at $2.5 \mathrm{ps}$ and $30 \mathrm{fs}$ (black). The negative bands seen for an $\mathrm{eBE}$ of $3-8 \mathrm{eV}$ are primarily associated with the excited state of $\mathrm{CHD}$, and the positive bands at $8-11 \mathrm{eV}$ are due to reaction products. The intensity above $11 \mathrm{eV}$ 
appears weaker because signals due to the excited states of CHD and the reaction products largely cancel each other. The spectra overlain on the observed spectrum are the predicted spectra for CHD and HT reaction products at 2 ps after photoexcitation, and were obtained by semi-empirical CISD dynamical calculations. Static photoelectron spectra of cold CHD and HT( $t Z t)$ are shown along with the band positions predicted by XMS-CASPT2 quantum chemical calculations for CHD and three HT isomers. (B) Time profile of photoelectron intensities observed for eBE values of $8,8.4$ and $10 \mathrm{eV}$. Open symbols are the experimental data and smooth curves are the signal intensities, which are proportional to the population obtained by analysis using the scheme shown in Fig. 3(E). The oscillatory components were extracted as the difference between the experimental data and the simulated population of the reaction products. It is noted that the phase of the oscillations is shifted by $\pi$ between the traces at 8 and $10 \mathrm{eV}$. The quantum beats are damped faster at 10 than at $8 \mathrm{eV}$. The trace at $8.4 \mathrm{eV}$ is for the photoelectron band center of the ground-state CHD, for which no oscillatory component is identified. (C) Frequency components extracted from the oscillatory components using a Fourier transform. Frequencies were converted to energy and are presented in units $\mathrm{of}^{-1}$.

\section{Methods}

A one-box $1 \mathrm{kHz}$ Ti:sapphire regenerative amplifier (Astrella, $35 \mathrm{fs}, 800 \mathrm{~nm}, 1 \mathrm{kHz}, 6 \mathrm{~mJ}$ ) was used as a driving laser for non-linear optical processes to generate the DUV pump (267.5 nm) and XUV probe $(57.1 \mathrm{~nm})$ pulses. The XUV pulses were produced using high-harmonic generation in $\mathrm{Kr}$ gas with the second harmonic $(2 \omega, 0.29 \mathrm{~mJ})$ of the Ti:sapphire laser as a driving pulse. ${ }^{28}$ The $2 \omega$ laser pulses were produced with a $0.3-\mathrm{mm}$ thick beta barium borate (BBO) crystal, and focused into a $\mathrm{Kr}$ gas cell using a quartz lens $(\mathrm{f}=500 \mathrm{~mm})$. The 14 th order $(14 \omega: 57 \mathrm{~nm}, 21.7 \mathrm{eV})$ single harmonic was selected with a grating-based time-preserving monochromator. The DUV pump pulses were generated by a filamentation four-wave mixing light source in Ar gas using the $\omega(0.5 \mathrm{~mJ})$ and $2 \omega(0.3 \mathrm{~mJ})$ pulses. ${ }^{36}$ The multi-color output from the filamentation cell was reflected and monochromatized using multilayer dielectric mirrors for $3 \omega$ in a vacuum chamber. The timing of the $3 \omega$ pulses was controlled 
by a vacuum-compatible translation stage with $5-\mathrm{nm}$ resolution. The pulse width and energy of the pump beam were sub-30 fs and $800 \mathrm{~nJ} /$ pulse, respectively. Since the entire optical path was kept under vacuum and no transmitting optical components were used, material dispersion was minimized. The cross correlation between the pump and probe pulses was determined to be 48 fs using non-resonant ionization of Xe.

The sample of 1,3-cyclohexadiene (CHD) was seeded by a He career gas and injected into a photoelectron spectrometer through a pinhole $(\varnothing 0.1 \mathrm{~mm})$ with a stagnation pressure of $0.06 \mathrm{MPa}$ at room temperature. The PKE distribution was measured using a magnetic bottle time-of-flight spectrometer. ${ }^{45}$ The photoelectrons traveled through a $1300-\mathrm{mm}$-long flight tube and were detected using a microchannel plate detector (MCP, $\varnothing 42 \mathrm{~mm}$ ) mounted at the end of the flight tube. A retardation voltage of $-6.0 \mathrm{~V}$ was applied to the flight tube in order to reject low-energy electrons produced by one-color two-photon ionization by the pump pulses. The measured PKE was calibrated using the photoelectron bands of ${ }^{2} \mathrm{P}_{3 / 2}$ and ${ }^{2} \mathrm{P}_{1 / 2}$ of $\mathrm{Xe}$. The energy resolution was estimated to be $0.12 \mathrm{eV}$. The pressure in the photoionization chamber and TOF analyzer were $4.0 \times 10^{-5}$ and $<1.0 \times 10^{-7}$ Torr, respectively, during the experiment.

\section{AUTHOR INFORMATION}

\section{Corresponding Author}

*suzuki@kuchem.kyoto-u.ac.jp

\section{Present Address}

'Department of Chemistry, Faculty of Science, Kyushu University, 744 Motooka, Nishi-ku, Fukuoka 819-0395, Japan

\section{Notes}

The authors declare no competing financial interests.

\section{ACKNOWLEDGMENT}


This work was supported by JSPS KAKENHI Grant No. 15H05753. M.K. gratefully acknowledges fruitful discussions with Professor F. Remacle and Dr. B. Mignolet on electronic structure computations and the support from the Japan-Belgium Research Cooperative Program between JSPS and F.R.S.-FNRS (Grant No. JPJSBP120192201).

\section{References}

1. Deb S, Weber PM. The ultrafast pathway of photon-induced electrocyclic ring-opening reactions: The case of 1,3-cyclohexadiene. Annual Review of Physical Chemistry 2011, 62: 19-39.

2. Arruda BC, Sension RJ. Ultrafast polyene dynamics: The ring opening of 1,3-cyclohexadiene derivatives. Physical Chemistry Chemical Physics 2014, 16(10): 4439-4455.

3. Holick MF. Vitamin-D - new horizons for the 21 st-century. American Journal of Clinical Nutrition 1994, 60(4): 619-630.

4. Woodward RB, Hoffmann R. The Conservation of Orbital Symmetry. Angew Chem Int Edit 1969, 8: $781-932$.

5. Garavelli M, Page CS, Celani P, Olivucci M, Schmid WE, Trushin SA, et al. Reaction path of a sub200 fs photochemical electrocyclic reaction. Journal of Physical Chemistry A 2001, 105(18): 44584469.

6. van der Lugt WT, Oosterhoff LJ. Quantum-chemical interpretation of photo-induced electrocyclic reactions. Chem Commun 1968(20): 1235-+.

7. Kosma K, Trushin SA, Fuss W, Schmid WE. Cyclohexadiene ring opening observed with 13 fs resolution: coherent oscillations confirm the reaction path. Physical Chemistry Chemical Physics 2009, 11(1): 172-181.

8. Fuß W, Schmid WE, Trushin SA. Time-resolved dissociative intense-laser field ionization for probing dynamics: Femtosecond photochemical ring opening of 1,3-cyclohexadiene. Journal of Chemical Physics 2000, 112(19): 8347-8362.

9. Celani P, Bernardi F, Robb MA, Olivucci M. Do photochemical ring-openings occur in the spectroscopic state? ${ }^{1} \mathrm{~B}_{2}$ pathways for the cyclohexadiene/hexatriene photochemical interconversion. 
Journal of Physical Chemistry 1996, 100(50): 19364-19366.

10. Tamura $\mathrm{H}$, Nanbu $\mathrm{S}$, Ishida $\mathrm{T}$, Nakamura $\mathrm{H}$. $\mathrm{Ab}$ initio nonadiabatic quantum dynamics of cyclohexadiene/hexatriene ultrafast photoisomerization. Journal of Chemical Physics 2006, 124(8).

11. Mori T, Kato S. Dynamic electron correlation effect on conical intersections in photochemical ringopening reaction of cyclohexadiene: MS-CASPT2 study. Chemical Physics Letters 2009, 476(1-3): 97100.

12. Nenov A, Kölle P, Robb MA, de Vivie-Riedle R. Beyond the van der Lugt/Oosterhoff Model: When the conical intersection Seam and the $\mathrm{S}_{1}$ minimum energy path do not cross. J Org Chem 2010, 75(1): 123129.

13. Schönborn JB, Sielk J, Hartke B. Photochemical ring-opening of cyclohexadiene: Quantum wavepacket dynamics on a global ab Initio potential energy surface. Journal of Physical Chemistry A 2010, 114(12): 4036-4044.

14. White JL, Kim J, Petrović VS, Bucksbaum PH. Ultrafast ring opening in 1,3-cyclohexadiene investigated by simplex-based spectral unmixing. Journal of Chemical Physics 2012, 136(5).

15. Ohta A, Kobayashi O, Danielache SO, Nanbu S. Nonadiabatic ab initio molecular dynamics of photoisomerization reaction between 1,3-cyclohexadiene and 1,3,5-cis-hexatriene. Chemical Physics 2015, 459: 45-53.

16. Lei YB, Wu HY, Zheng XL, Zhai GH, Zhu CY. Photo-induced 1,3-cyclohexadiene ring opening reaction: Ab initio on-the-fly nonadiabatic molecular dynamics simulation. Journal of Photochemistry and Photobiology A-Chemistry 2016, 317: 39-49.

17. Tudorovskaya M, Minns RS, Kirrander A. Effects of probe energy and competing pathways on timeresolved photoelectron spectroscopy: The ring-opening of 1,3-cyclohexadiene. Physical Chemistry Chemical Physics 2018, 20(26): 17714-17726.

18. Polyak I, Hutton L, Crespo-Otero R, Barbatt M, Knowles PJ. Ultrafast photoinduced dynamics of 1,3cyclohexadiene using XMS-CASPT2 surface hopping. Journal of Chemical Theory and Computation 2019, 15(7): 3929-3940.

19. Filatov M, Min SK, Kim KS. Non-adiabatic dynamics of ring opening in cyclohexa-1,3-diene described by an ensemble density-functional theory method. Molecular Physics 2019, 117(9-12): 1128-1141. 
20. Kuthirummal N, Rudakov FM, Evans CL, Weber PM. Spectroscopy and femtosecond dynamics of the ring opening reaction of 1,3-cyclohexadiene. Journal of Chemical Physics 2006, 125(13).

21. Minitti MP, Budarz JM, Kirrander A, Robinson JS, Ratner D, Lane TJ, et al. Imaging Molecular Motion: Femtosecond X-Ray Scattering of an Electrocyclic Chemical Reaction. Phys Rev Lett 2015, 114(25).

22. Adachi S, Sato M, Suzuki T. Direct observation of ground-state product formation in a 1,3cyclohexadiene ring-opening reaction. Journal of Physical Chemistry Letters 2015, 6(3): 343-346.

23. Pemberton CC, Zhang Y, Saita K, Kirrander A, Weber PM. From the (1B) spectroscopic state to the photochemical product of the ultrafast ring-opening of 1,3-cyclohexadiene: A spectral observation of the complete reaction path. Journal of Physical Chemistry A 2015, 119(33): 8832-8845.

24. Iikubo R, Sekikawa T, Harabuchi Y, Taketsugu T. Structural dynamics of photochemical reactions probed by time-resolved photoelectron spectroscopy using high harmonic pulses. Faraday Discuss 2016, 194: 147-160.

25. Schalk O, Geng T, Thompson T, Baluyot N, Thomas RD, Tapavicza E, et al. Cyclohexadiene revisited: A time-resolved photoelectron spectroscopy and ab initio study. Journal of Physical Chemistry A 2016, 120(15): 2320-2329.

26. Attar AR, Bhattacherjee A, Pemmaraju CD, Schnorr K, Closser KD, Prendergast D, et al. Femtosecond x-ray spectroscopy of an electrocyclic ring-opening reaction. Science 2017, 356(6333): 54-58.

27. Wolf TJA, Sanchez DM, Yang J, Parrish RM, Nunes JPF, Centurion M, et al. The photochemical ringopening of 1,3-cyclohexadiene imaged by ultrafast electron diffraction. Nat Chem 2019, 11(6): 504509.

28. Nishitani J, Yamamoto Y, West CW, Karashima S, Suzuki T. Binding energy of solvated electrons and retrieval of true UV photoelectron spectra of liquids. Sci Adv 2019, 5(8): 7.

29. Krause JL, Schafer KJ, Kulander KC. High-order harmonic generation from atoms and ions in the high intensity regime. Physical Review Letters 1992, 68(24): 3535-3538.

30. Corkum PB. Plasma Perspective on Strong-Field Multiphoton Ionization. Phys Rev Lett 1993, 71(13): 1994-1997. 
31. Lewenstein M, Balcou P, Ivanov MY, L'huillier A, Corkum PB. Theory of high-harmonic generation by low-frequency laser fields. Physical Review A 1994, 49(3): 2117-2132.

32. Corkum PB, Krausz F. Attosecond science. Nature Physics 2007, 3(6): 381-387.

33. Hentschel M, Kienberger R, Spielmann C, Reider GA, Milosevic N, Brabec T, et al. Attosecond metrology. Nature 2001, 414(6863): 509-513.

34. Krausz F, Ivanov M. Attosecond physics. Rev Mod Phys 2009, 81(1): 163-234.

35. Galli M, Wanie V, Lopes DP, Mansson EP, Trabattoni A, Colaizzi L, et al. Generation of deep ultraviolet sub-2-fs pulses. Opt Lett 2019, 44(6): 1308-1311.

36. Fuji T, Horio T, Suzuki T. Generation of $12 \mathrm{fs}$ deep-ultraviolet pulses by four-wave mixing through filamentation in neon gas. Optics Letters 2007, 32(17): 2481-2483.

37. Granovsky AA. Extended multi-configuration quasi-degenerate perturbation theory: The new approach to multi-state multi-reference perturbation theory. Journal of Chemical Physics 2011, 134(21): 14.

38. Autrey D, Choo J, Laane J. Spectroscopic determination of the ring-twisting potential energy function of 1,3-cyclohexadiene and comparison with ab initio calculations. Journal of Physical Chemistry A 2001, 105(45): 10230-10236.

39. Johnson PJM, Halpin A, Morizumi T, Prokhorenko VI, Ernst OP, Miller RJD. Local vibrational coherences drive the primary photochemistry of vision. Nat Chem 2015, 7(12): 980-986.

40. Schoenlein RW, Peteanu LA, Mathies RA, Shank CV. The 1st step in vision - femtosecond isomerization of rhodopsin. Science 1991, 254(5030): 412-415.

41. Kukura P, McCamant DW, Yoon S, Wandschneider DB, Mathies RA. Structural observation of the primary isomerization in vision with femtosecond-stimulated Raman. Science 2005, 310(5750): 10061009.

42. Schapiro I, Ryazantsev MN, Frutos LM, Ferre N, Lindh R, Olivucci M. The Ultrafast Photoisomerizations of Rhodopsin and Bathorhodopsin Are Modulated by Bond Length Alternation and HOOP Driven Electronic Effects. J Am Chem Soc 2011, 133(10): 3354-3364.

43. Schnedermann C, Liebel M, Kukura P. Mode-Specificity of Vibrationally Coherent Internal Conversion 
in Rhodopsin during the Primary Visual Event. $J$ Am Chem Soc 2015, 137(8): 2886-2891.

44. Hoffman DP, Mathies RA. Femtosecond Stimulated Raman Exposes the Role of Vibrational Coherence in Condensed-Phase Photoreactivity. Accounts Chem Res 2016, 49(4): 616-625.

45. Kruit P, Read FH. Magnetic-field parallelizer for $2 \pi$ electron-spectrometer and electron-image magnifier. J Phys E Sci Instrum 1983, 16(4): 313-324. 


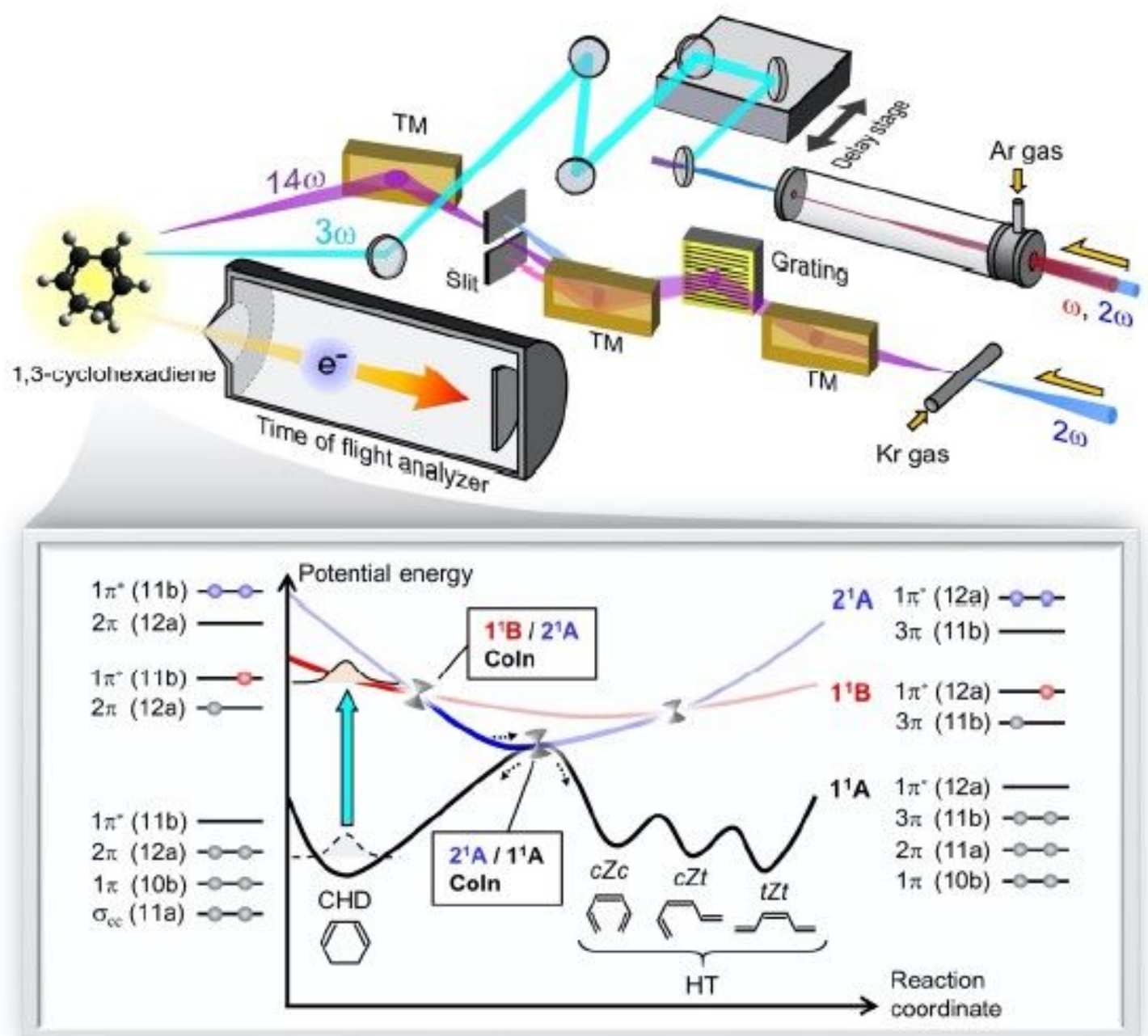

\section{Figure 1}

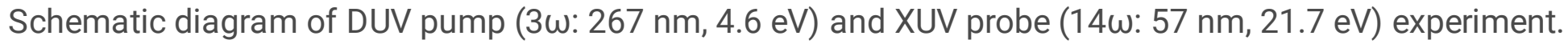
(TM: toroidal mirror, Coln: conical intersection, CHD: 1,3-cyclohexadiene, HT: 1,3,5-hexatriene) 

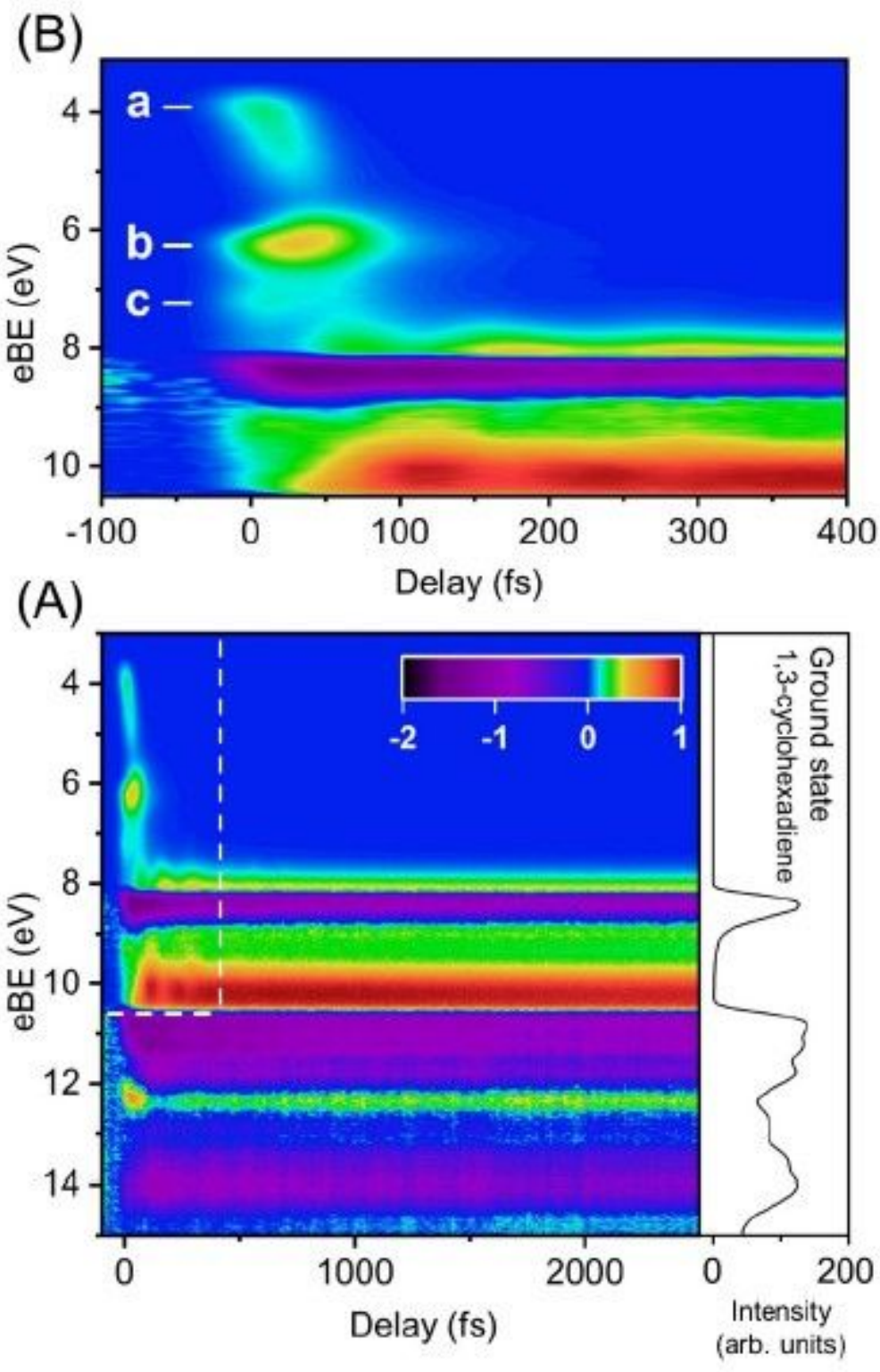

\section{Figure 2}

(A) Two-dimensional map of the photoelectron spectra measured as a function of the pump-probe delay time, with the photoelectron spectrum of the ground-state CHD shown on the right. The one-color photoionization signal from unexcited $\mathrm{CHD}$ has already been subtracted; therefore, the negative signals shown in violet correspond to signals due to photoexcitation depletion by the pump pulses. (B) Blowup of the map at short delay times. Photoionization from the $11 \mathrm{~B}$ to the cationic ground state $12 \mathrm{~A}$ and the $12 \mathrm{~B}$ and 22B (or 32B) excited states is indicated by a, b and c, respectively. 

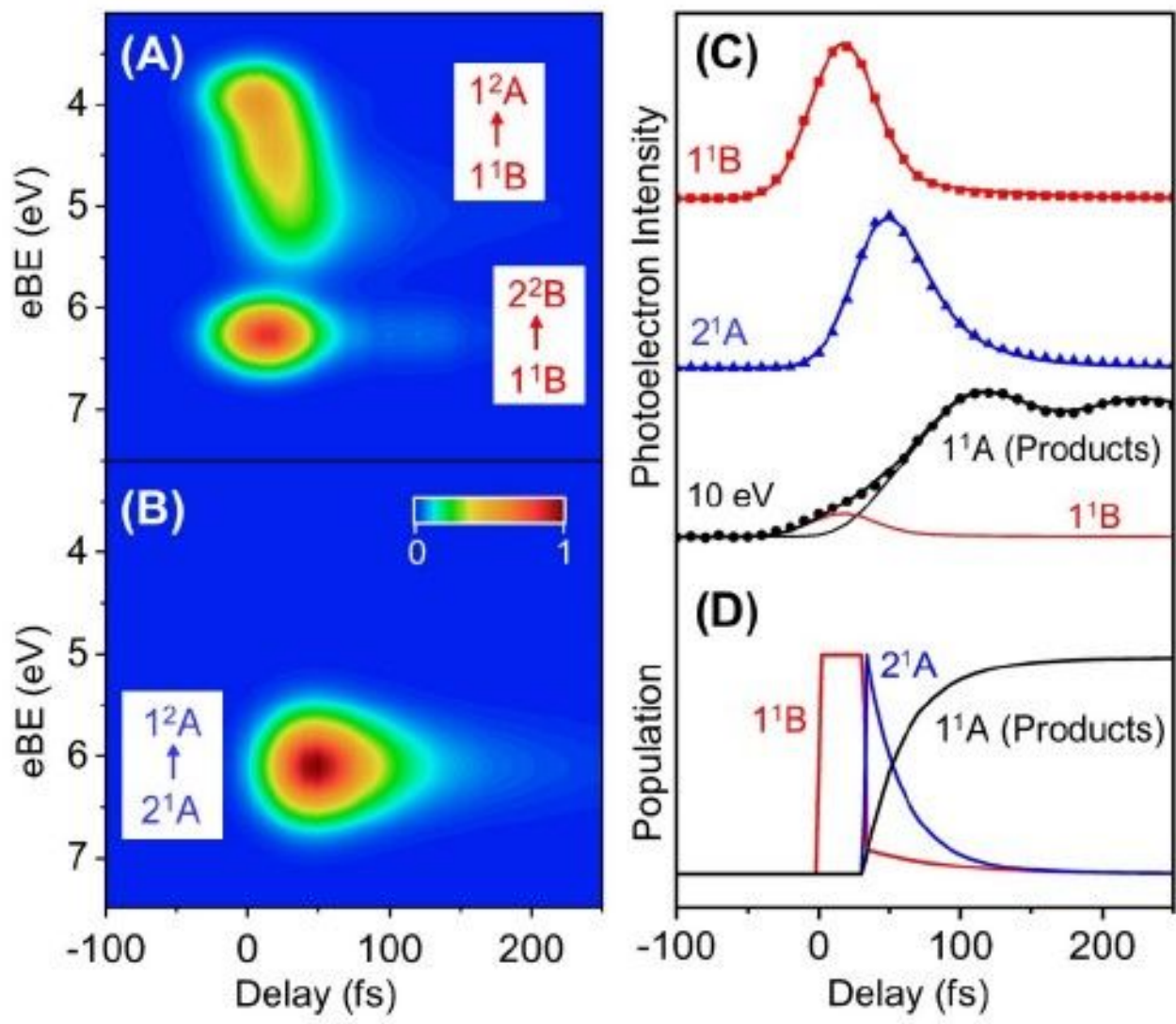

(E)

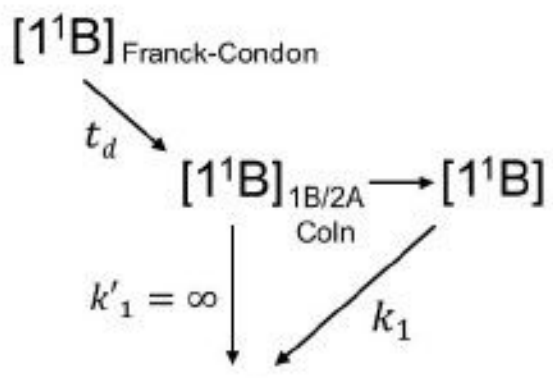

$\left[2^{1} \mathrm{~A}\right]$

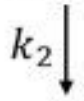

[11'A]

\section{Figure 3}

Photoelectron spectra of (A) 11B and (B) 21A separated using least-squares fitting with the kinetics scheme shown in (E). (C) Time profiles of photoelectron intensities. Dots and solid lines show the experimental data and fitting results, respectively. Red and blue represent ionization from the 11B and $21 \mathrm{~A}$ states, respectively, while black represents the integrated photoelectron intensity in the eBE range of 9.9-10.0 eV, which is predominantly due to reaction products. (D) Population in each electronic state calculated by deconvoluting with the experimental time resolution. (E) Kinetic scheme employed for the analysis. Bifurcation of the dynamics via non-adiabatic transitions at the $11 \mathrm{~B} / 21 \mathrm{~A}$ conical intersection is taken into account. See Supporting Information for more details of our analysis. 

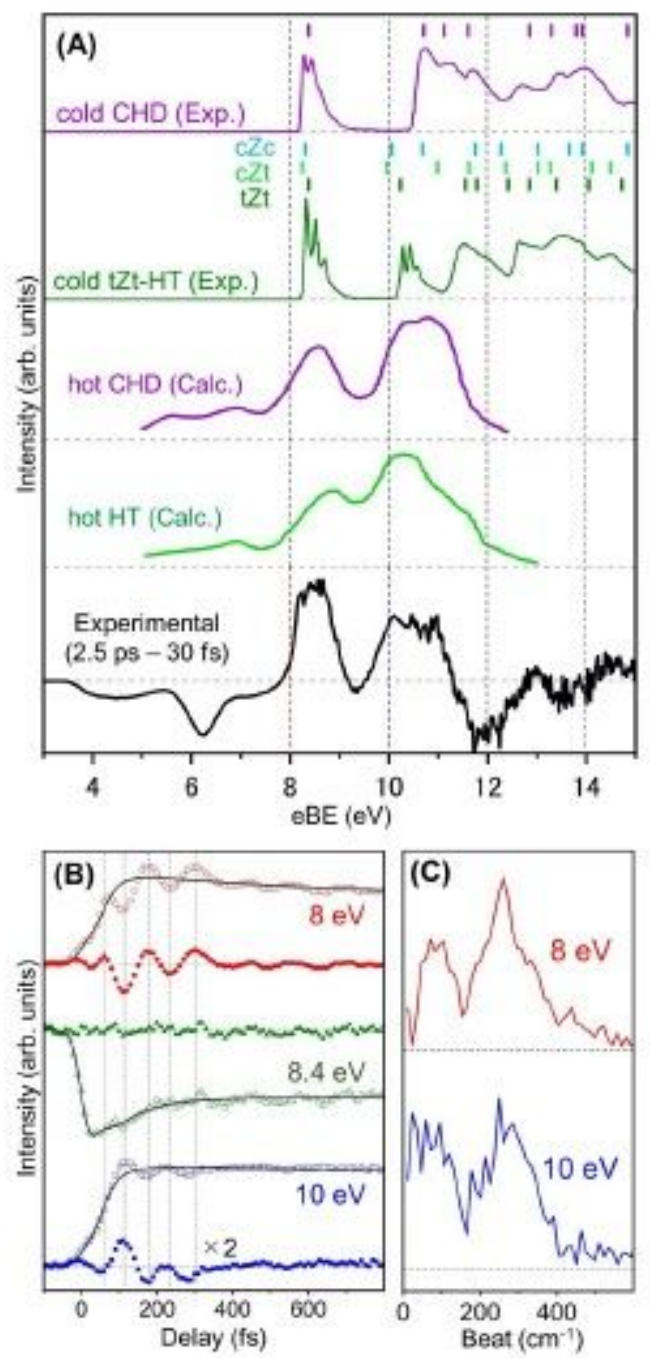

Figure 4

(A) Difference between transient photoelectron spectra measured at $2.5 \mathrm{ps}$ and $30 \mathrm{fs}$ (black). The negative bands seen for an $\mathrm{eBE}$ of 3-8 $\mathrm{eV}$ are primarily associated with the excited state of $\mathrm{CHD}$, and the positive bands at $8-11 \mathrm{eV}$ are due to reaction products.

\section{Supplementary Files}

This is a list of supplementary files associated with this preprint. Click to download.

- Supplementaryinformationsuzuki.pdf

- FigS1.jpg

- FigS2.jpg

- FigS3.jpg

- FigS4.jpg

- FigS5.jpg 
- FigS6.jpg

- FigS7.jpg

- FigS8.jpg

- FigS9.jpg

- FigS10.jpg

- FigS11.jpg

- FigS12.jpg

- Tables1.png

- Tables2.png

- Tables3.png

- Tables4.png

- TableS5.png

- TableS6.png

- TableS7.png 